\title{
In-Hospital Outcomes and Trends of Tricuspid Valve Surgery in Heart Transplant Patients
}

\author{
Moghniuddin Mohammed ${ }^{a} \quad$ Aniket S. Ralia ${ }^{a}$ Tyler Buechler ${ }^{a}$ \\ Venkat Vuddanda $^{b}$ Juwairiya Arshi ${ }^{a}$ Seyed Hamed Hosseini Dehkordi ${ }^{a}$ \\ Jonathan Chandler ${ }^{a}$ Robert Weidling ${ }^{a}$ Travis Abicht ${ }^{c}$ \\ Nicholas Haglund ${ }^{a} \quad$ Andrew Sauer $^{a}$ Zubair Shah ${ }^{a}$ \\ a Department of Cardiovascular Diseases, The University of Kansas Health System, Kansas \\ City, MO, USA; ${ }^{b}$ Division of General Internal Medicine, Brigham and Women's Hospital, \\ Boston, MA, USA; 'Department of Cardiothoracic Surgery, The University of Kansas Health \\ System, Kansas City, MO, USA
}

\section{What Is It about?}

Our study is a retrospective analysis of the National Inpatient Sample (NIS) looking at trends and outcomes of tricuspid valve (TV) surgery among heart transplant recipients. TV insufficiency is not uncommon following heart transplantation, perhaps due to continued trauma to the valve from frequent endomyocardial biopsies. However, there remains a paucity of literature on the trends and outcomes of this condition, especially in regard to postsurgical interventions. Our analysis of the NIS database provides insight into trends in contemporary management and outcomes of TV insufficiency in the USA.

\section{Keywords}

Tricuspid valve surgery $\cdot$ Heart transplant · Outcomes · Nationwide Inpatient Sample database

\section{Abstract}

Introduction: Tricuspid valve (TV) regurgitation is the most common valvular pathology after orthotopic heart transplant (OHT). The number of transplants being performed is increasing with patients living longer after heart transplant. Data on TV surgery in OHT recipients is limited. Methods: We sought to analyze the outcomes of patients undergoing TV surgery from a large diverse, multicentric, nationwide cohort using the Nationwide Inpatient Sample (NIS)

Moghniuddin Mohammed and Aniket S. Rali contributed equally to this paper. 
database. Results: A total of 42,766 TV repair or replacement (bioprosthetic and mechanical) involving adult patients (age $\geq 18$ years) between 2007 and September 2015 were identified. Of these, 366 were performed in patients with OHT. TV repair was the most common surgery performed in both groups (OHT group and native heart group). Compared to the native group, patients with $\mathrm{OHT}$ had a significantly higher incidence of cardiogenic shock (20 vs. $11 \%$, $p=0.024)$, acute kidney injury (AKI) (59 vs. $30 \%, p<0.001)$, and $\mathrm{AKI}$ requiring hemodialysis (13 vs. $4 \%, p<0.001$ ). Also, the mean length of stay for the index admission was significantly longer in the OHT group ( 27 vs. 17 days, $p=0.008$ ). The mortality rate was similar between the two groups ( $7 \mathrm{vs.} 8 \%, p=0.753$ ). The number of TV surgeries performed in OHT patients from 2007 to 2014 have remained stable ( $p=0.803)$ compared to those in native heart patients which showed a significantly increasing trend $(p=0.019)$ during the same time period. Conclusions: TV surgery remains an important treatment modality among the OHT population and carries a similar mortality during index hospitalization as that in native heart patients undergoing TV surgery.

(C) 2020 The Author(s)

Published by S. Karger AG, Basel

\section{Introduction}

The most common valvular pathology after orthotopic heart transplant (OHT) is tricuspid valve (TV) regurgitation, with a reported incidence between 19 and 84\% [1]. The two most common factors contributing to the development of TV regurgitation after OHT are operative technique (bi-caval vs. bi-atrial) and frequent post-transplant endomyocardial biopsies [1]. While the majority of TV regurgitations following OHT are mild and clinically insignificant, it is estimated that approximately $34 \%$ lie in the moderate-to-severe range [2]. Up to $5.8 \%$ of patients develop refractory symptoms that warrant surgical correction [3]. TV regurgitation after OHT is associated with adverse clinical outcomes including right heart dysfunction, renal impairment, and mortality as high as $62.5 \%[2,4-7]$. The role of prophylactic donor TV repair in reducing post-transplant TV regurgitation and death remains unclear with small studies showing mixed results [8-12]. Furthermore, reporting of surgical outcomes among patients treated for post-OHT TV regurgitation is limited to small single-center experiences $[8,13]$.

\section{Methods}

Data Source

We conducted a retrospective review of patients undergoing TV surgery. We analyzed discharge data from the Nationwide Inpatient Sample (NIS) between 2007 and September 2015. The NIS, developed by the Agency for Healthcare Research and Quality as part of the Healthcare Cost and Utilization Project (HCUP), is the largest all-payer inpatient database in the USA. The NIS contains a $20 \%$ stratified sample of all discharges from US nonfederal shortterm general hospitals, subspecialty hospitals, and public hospitals, which are stratified based on the number of beds, ownership, hospital teaching status, US region, and the state. This stratified random sampling ensures that the database is representative of the US population and accounts for $90 \%$ of all hospitalizations in the USA.

\section{Study Population}

Based on the International Classification of Diseases, Ninth Revision (ICD-9), clinical modification codes in the individual discharge records (online suppl. Table1; for all online 
Mohammed et al.: In-Hospital Outcomes and Trends of TV Surgery in Heart Transplant Patients

Fig. 1. Flow diagram showing patient selection. TV, tricuspid valve.

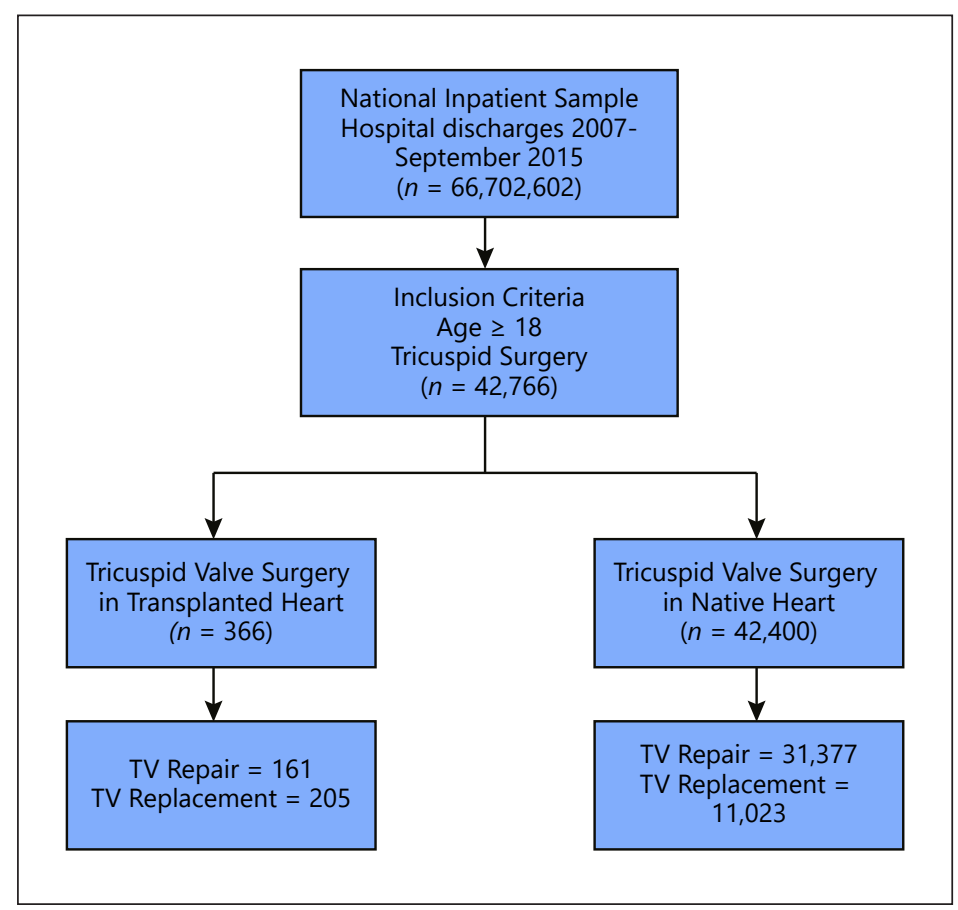

suppl. material, see www.karger.com/doi/10.1159/000507179), adult patients (age $\geq 18$ years) who underwent TV repair or replacement were identified. They were divided into two groups based on the presence of codes related to history of or complications related to OHT (OHT group and native heart group). Patients were further stratified based on the type of TV surgery performed, which included TV repair, bioprosthetic TV replacement, and mechanical TV replacement.

\section{Outcomes}

The main outcomes assessed were the number of surgeries performed annually in the two groups, type of surgery performed, concomitant cardiac surgeries performed, in-hospital mortality, postoperative morbidity, length of stay (LOS), and total hospitalization charges.

\section{Statistical Analysis}

Descriptive statistics are presented as mean \pm standard deviation for continuous variables and categorical data are presented as percentages. Baseline characteristics were compared between the two groups using either the $t$ test or the Wilcoxon rank-sum test, as deemed appropriate, for continuous variables. The categorical variables were compared using svychisq (performs second-order Rao-Scott correction to Pearson $\chi^{2}$ test and computes $p$ values based on Satterthwaite approximation) or the Fisher exact test, as deemed appropriate. The Mann-Kendall test was used for yearly trend analysis and only data from 2007 through 2014 was analyzed. Data for the calendar year 2015 contains both ICD-9 and ICD-10 codes which makes annual trend analysis challenging and thus was not included in the trend analysis.

Analysis was performed using R software version 3.5.3 [14] R studio interface on a Windows x86_64 machine. We used the "tableone" package for $\mathrm{R}$ to generate all the tables and the "Survey" package version 3.35.1 to set up survey design and obtain national estimates accounting for the complex sample design of NIS. The "Kendall" package version 2.2 was used for yearly trend analysis. $p<0.05$ was required for statistical significance. 
Table 1. Baseline characteristics

\begin{tabular}{|c|c|c|c|}
\hline Variables & $\begin{array}{l}\text { OHT } \\
(n=366)\end{array}$ & $\begin{array}{l}\text { Native heart } \\
(n=42,400)\end{array}$ & $p$ values \\
\hline Age, years & $52 \pm 15.02$ & $61 \pm 17.05$ & $<0.001$ \\
\hline Female gender & $140(38)$ & $24,442(58)$ & 0.002 \\
\hline Race & & & 0.235 \\
\hline White & $206(68)$ & $25,280(71)$ & \\
\hline Black & $68(22)$ & $4,676(13)$ & \\
\hline Primary expected payer & & & 0.01 \\
\hline Medicaid & $38(10)$ & 5,707 (13) & \\
\hline Medicare & $176(48)$ & $23,159(55)$ & \\
\hline No charge & 0 & $230(1)$ & \\
\hline Other & $34(9)$ & $971(2)$ & \\
\hline Private & $119(32)$ & $10,882(26)$ & \\
\hline Self-pay & 0 & $1,379(3)$ & \\
\hline Median household income & & & 0.974 \\
\hline $0-25$ th percentile & $93(26)$ & $10,933(26)$ & \\
\hline 26-50th percentile & $83(23)$ & $10,447(25)$ & \\
\hline 51st-75th percentile & $91(26)$ & $10,417(25)$ & \\
\hline 76th-100th percentile & $88(25)$ & $9,482(23)$ & \\
\hline Teaching status & 357 (99) & $33,332(79)$ & $<0.001$ \\
\hline Hypertension & $197(54)$ & $21,564(51)$ & 0.6 \\
\hline Diabetes mellitus & $89(24)$ & $8,942(21)$ & 0.432 \\
\hline Coronary artery disease & 69 (19) & $14,745(35)$ & 0.003 \\
\hline Cirrhosis & $19(5)$ & $704(2)$ & 0.013 \\
\hline History of pacemaker/defibrillator & $20(5)$ & $3,835(9)$ & 0.366 \\
\hline Obesity & $29(8)$ & $4,950(12)$ & 0.363 \\
\hline Valvular heart disease & $9(3)$ & $2,515(6)$ & 0.22 \\
\hline Peripheral vascular disease & $25(7)$ & $3,810(9)$ & 0.474 \\
\hline Chronic heart failure & $4(1)$ & $2,250(5)$ & 0.099 \\
\hline Chronic lung disease & $34(9)$ & $7,826(18)$ & 0.031 \\
\hline Chronic liver disease & $19(5)$ & $2,217(5)$ & 0.97 \\
\hline Chronic kidney disease & $208(57)$ & $8,856(21)$ & $<0.001$ \\
\hline Coagulopathy & $121(33)$ & $13,844(33)$ & 0.936 \\
\hline Prior valve & $10(3)$ & $1,407(3)$ & 0.773 \\
\hline Prior CABG & $5(1)$ & $1,751(4)$ & 0.238 \\
\hline Prior VAD/TAH & $15(4)$ & $30(0.07)$ & $<0.001$ \\
\hline Charlson comorbidity index $\geq 3$ & $216(59)$ & $15,292(36)$ & $<0.0001$ \\
\hline
\end{tabular}

Data are presented as $n(\%)$ or mean \pm SD, as appropriate.

\section{Results}

A total of 42,766 hospital admissions with the procedure code of TV surgery were identified between 2007 and September 2015. Patients who had codes listed for history of or complications related to OHT accounted for 366 cases of TV surgery (Fig. 1).

Between the two groups, patients with native hearts were more likely to be older (61 vs. 51 years, $p<0.001$ ) and female (58 vs. 38\%, $p=0.002$ ), and less likely to have their TV intervention at an academic teaching hospital ( 79 vs. $99 \%, p<0.001$; Table 1). Patients with prior OHT had a lower prevalence of coronary artery disease (19 vs. $35 \%, p=0.003$ ) and chronic lung disease ( 9 vs. $18 \%, p=0.031$ ). However, patients with prior OHT had a higher prevalence of cirrhosis ( 5 vs. $2 \%, p=0.013$ ), chronic kidney disease (57 vs. $12 \%, p<0.001$ ), and prior ventricular assistance device ( 4 vs. $0 \%, p<0.001)$. Significantly higher numbers of patients 
Table 2. Concomitant surgeries

\begin{tabular}{lccc}
\hline Variables & $\begin{array}{l}\text { OHT } \\
(n=366)\end{array}$ & $\begin{array}{l}\text { Native heart } \\
(n=42,400)\end{array}$ & $p$ values \\
\hline $\begin{array}{l}\text { Isolated TV surgery } \\
\text { Surgery type }\end{array} \quad 215(59)$ & $9,621(23)$ & $<0.0001$ \\
$\quad$ TV repair & $161(44)$ & $31,377(74)$ & $<0.001$ \\
$\quad$ TV replacement - bioprosthetic valve & $146(40)$ & $6,066(14)$ & \\
$\quad$ TV replacement - mechanical valve & $59(16)$ & $4,958(12)$ & \\
Concomitant mitral valve surgery & $25(7)$ & $24,371(57)$ & $<0.001$ \\
Concomitant aortic valve surgery & $10(3)$ & $8,467(20)$ & $<0.001$ \\
Concomitant pulmonic valve surgery & 0 & $890(2)$ & $<0.001$ \\
Concomitant other valve surgeries & $20(5)$ & $3,138(7)$ & 0.515 \\
Concomitant CABG & $14(4)$ & $8,269(20)$ & $<0.001$ \\
Concomitant heart transplant & $93(25)$ & $116(0.27)$ & $<0.001$ \\
Concomitant other cardiac surgery & $48(13)$ & $2,096(5)$ & 0.002 \\
\hline
\end{tabular}

Data are presented as $n(\%)$.

Table 3. Outcomes of TV surgery stratified by heart transplant status

\begin{tabular}{lccr}
\hline Variables & \multicolumn{1}{l}{$\begin{array}{l}\text { OHT } \\
(n=366)\end{array}$} & $\begin{array}{l}\text { Native heart } \\
(n=42,400)\end{array}$ & $p$ values \\
\hline In-hospital mortality & $24(7)$ & $3,249(8)$ & 0.753 \\
Pacemaker placement & $20(5)$ & $4,618(11)$ & 0.202 \\
Blood transfusion & $96(26)$ & $15,538(37)$ & 0.078 \\
Cardiogenic shock & $72(20)$ & $4,756(11)$ & 0.024 \\
Postoperative infection & $9(2)$ & $543(1)$ & 0.371 \\
CVA or TIA & $5(1)$ & $1,547(4)$ & 0.286 \\
AKI & $217(59)$ & $12,794(30)$ & $<0.001$ \\
AKI requiring HD & $48(13)$ & $1,821(4)$ & $<0.001$ \\
LOS, days & $27.24 \pm 32.35$ & $17.55 \pm 17.08$ & 0.008 \\
Total charges, USD & $396,533 \pm 451,460$ & $297,735 \pm 288,091$ & 0.070 \\
Discharge & & & 0.173 \\
$\quad$ Home/self-care & $174(48)$ & $13,096(31)$ & \\
$\quad$ SNF/other facility & $67(18)$ & $12,139(29)$ & \\
\multicolumn{1}{l}{ Home health care } & $100(27)$ & $12,963(31)$ & \\
\hline
\end{tabular}

Data are presented as $n(\%)$ or mean $\pm \mathrm{SD}$, as appropriate.

in the OHT group had a Charlson Comorbidity Index (CCI) $\geq 3$ compared to patients who did not have an OHT (59 vs. $36 \%, p<0.001$ ).

In regard to TV surgery, patients with prior OHT had a significantly higher prevalence of isolated TV intervention compared to patients in the native heart group (59 vs. $23 \%, p<$ 0.0001; Table 2). Patients in the native heart cohort had a higher prevalence of concomitant mitral valve surgery ( 57 vs. $7 \%, p<0.001$ ), concomitant aortic valve surgery ( 20 vs. $3 \%, p<$ 0.001 ), and concomitant pulmonic valve surgery ( 2 vs. $0 \%, p<0.001)$. When stratified based on type of TV surgery, repair was the most common surgery performed in both groups. Among the two groups, patients with native heart had a greater number of tricuspid repair (74 vs. $44 \%, p<0.001$ ) and patients with OHT had a greater proportion of TV replacement with 
Table 4. Outcomes in OHT group stratified by type of TV surgery

\begin{tabular}{lcccc}
\hline Variables & $\begin{array}{l}\text { TV repair } \\
(n=161)\end{array}$ & $\begin{array}{l}\text { TV replacement }- \\
\text { bioprosthetic } \\
(n=146)\end{array}$ & $\begin{array}{l}\text { TV replacement - } \\
\text { mechanical } \\
(n=59)\end{array}$ & $p$ value \\
\hline In-hospital mortality & $15(9)$ & 0 & $10(17)$ & 0.143 \\
Pacemaker placement & 0 & $20(13)$ & 0 & 0.115 \\
Blood transfusion & $34(21)$ & $38(26)$ & $24(41)$ & 0.435 \\
Cardiogenic shock & $43(27)$ & $19(13)$ & $10(17)$ & 0.505 \\
Postoperative infection & $5(3)$ & $4(3)$ & 0 & 0.824 \\
CVA or TIA & 0 & 0 & $5(8)$ & 0.122 \\
AKI & $91(57)$ & $91(63)$ & $34(58)$ & 0.870 \\
AKI requiring HD & $5(3)$ & $24(17)$ & $19(33)$ & 0.024 \\
LOS, days & $34 \pm 41$ & $23 \pm 24$ & $18 \pm 14$ & 0.202 \\
Total charges, USD & $561,783 \pm 603,130$ & $280,431 \pm 291,418$ & $282,640 \pm 126,975$ & 0.432 \\
Discharge & $78(48)$ & $82(56)$ & $15(25)$ & \\
$\quad$ Home/self-care & $29(18)$ & $24(16)$ & $14(24)$ & \\
SNF/other facility & $40(25)$ & $40(28)$ & $20(34)$ & \\
Home health care & $15(9)$ & $0(0)$ & $10(17)$ & \\
\hline
\end{tabular}

Data are presented as $n(\%)$ or mean $\pm \mathrm{SD}$, as appropriate.

bioprosthetic valve ( 40 vs. $14 \%, p<0.001$ ) and mechanical valve (16 vs. $12 \%, p<0.05$ ) compared to patients with native hearts.

Table 3 summarizes complications for the overall group. Overall, the mortality rate was similar between the two groups ( 7 vs. $8 \%, p=0.753$ ). There was a significantly higher incidence of cardiogenic shock ( 20 vs. $11 \%, p=0.024$ ), acute kidney injury (AKI; 59 vs. $30 \%, p<$ 0.001 ), and AKI requiring hemodialysis (13 vs. $4 \%, p<0.001$ ) in the OHT group. Also, the mean LOS for the principal admission was significantly longer in the OHT group (27 vs. 17 days, $p=0.008$ ). There was no statistically significant difference between the two groups in regard to need for pacemaker implantation, blood transfusion, postoperative infection, CVA, or TIA.

Among the OHT cohort, there was no significant difference in complication rates between the tricuspid repair and replacement subgroups (Table 4). However, patients who underwent mechanical TV replacement were significantly more likely to develop AKI requiring hemodialysis compared to patients who underwent bioprosthetic replacement and TV repair (33 vs. 17 vs. $3 \%, p=0.024$ ). While the mortality rate was not significantly different amongst the three groups, the mechanical valve subgroup experienced $17 \%$ mortality rate, whereas the bioprosthetic valve subgroup did not have any in-hospital mortality.

We also analyzed the outcomes in the OHT group between isolated (only TV) and nonisolated TV surgery (Table 5). There were no significant differences between mortality and complication rates but LOS $(20 \pm 21.7$ vs. $30 \pm 28.7$ days, $p=0.006)$ and cost of hospitalization were significantly higher in the nonisolated TV surgery cohort $(611,051 \pm 592,665$ vs. $256,601 \pm 249,722, p=0.041$ ).

Yearly trend analysis showed that there was no significant change in volume of TV surgery ( $\tau=-0.109, p=0.803)$ and mortality $(\tau=-0.321, p=0.385)$ in 0HT patients. In contrast, the volume of surgery increased significantly in native heart patients $(\tau=0.714$, $p=0.019$ ) with improving mortality compared to the number of surgeries performed (Fig. 2, $3)$. 
Table 5. Outcomes in OHT group stratified by isolated versus nonisolated TV surgery

\begin{tabular}{lccc}
\hline Variables & $\begin{array}{l}\text { Isolated TV surgery } \\
(n=215)\end{array}$ & $\begin{array}{l}\text { Nonisolated TV } \\
\text { surgery }(n=151)\end{array}$ & $p$ value \\
\hline In-hospital mortality & $10(5)$ & $15(10)$ & 0.401 \\
Pacemaker placement & $20(9)$ & 0 & 0.165 \\
Blood transfusion & $67(31)$ & $29(19)$ & 0.329 \\
Cardiogenic shock & $29(13)$ & $43(29)$ & 0.153 \\
Postoperative infection & $4(2)$ & $5(3)$ & 0.781 \\
CVA or TIA & $5(2)$ & 0 & 0.409 \\
AKI & $116(54)$ & $101(67)$ & 0.265 \\
AKI requiring HD & $29(14)$ & $19(13)$ & 0.921 \\
LOS, days & $20 \pm 21.7$ & $30 \pm 28.7$ & 0.006 \\
Cost of hospitalization, USD & $256,601 \pm 249,722$ & $611,051 \pm 592,665$ & 0.041 \\
Discharge $\quad$ & & 0.724 \\
$\quad$ Home/self-care & $111(52)$ & $34(22)$ & \\
\multicolumn{1}{c}{ HomF/other facility } & $33(15)$ & $39(26)$ & \\
\hline
\end{tabular}

Data are presented as $n(\%)$ or mean \pm SD, as appropriate.

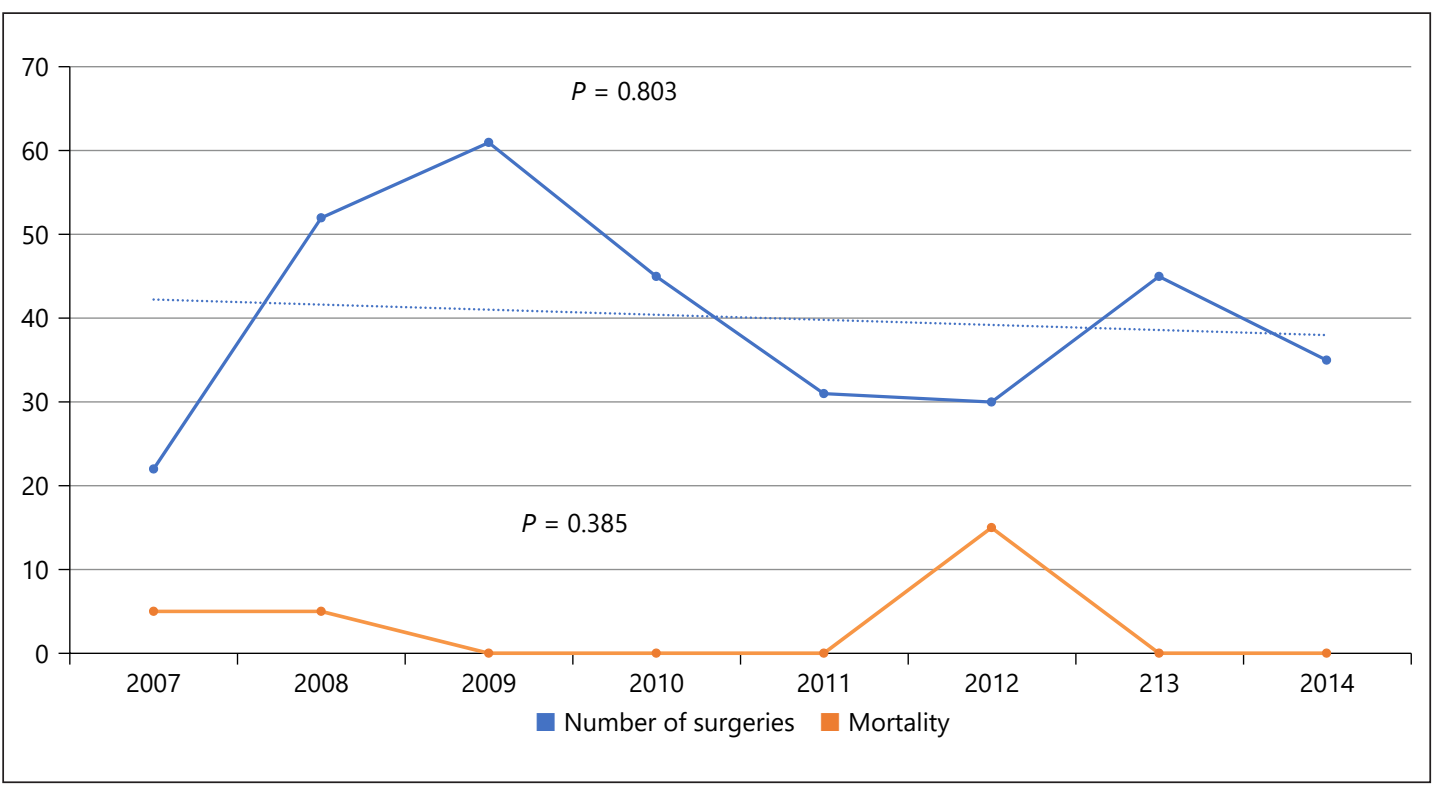

Fig. 2. Trends of tricuspid valve surgery and mortality in OHT.

\section{Discussion}

To the best of our knowledge, our study is the largest to date at reporting the trends and outcomes of TV surgery for TV regurgitation among OHT patients. We also compared outcomes after TV surgeries between native and transplanted hearts for the study period.

OHT patients undergoing TV surgeries are more likely to have a higher CCI score and a longer LOS during their index hospitalization than patients with native hearts, but their overall mortality is similar. The most common type of TV surgery among both of our study cohorts was TV repair, and patients with native hearts are more likely to have concomitant valve surgeries at the time of their TV operation. OHT patients undergoing TV replacement 
Mohammed et al.: In-Hospital Outcomes and Trends of TV Surgery in Heart Transplant Patients

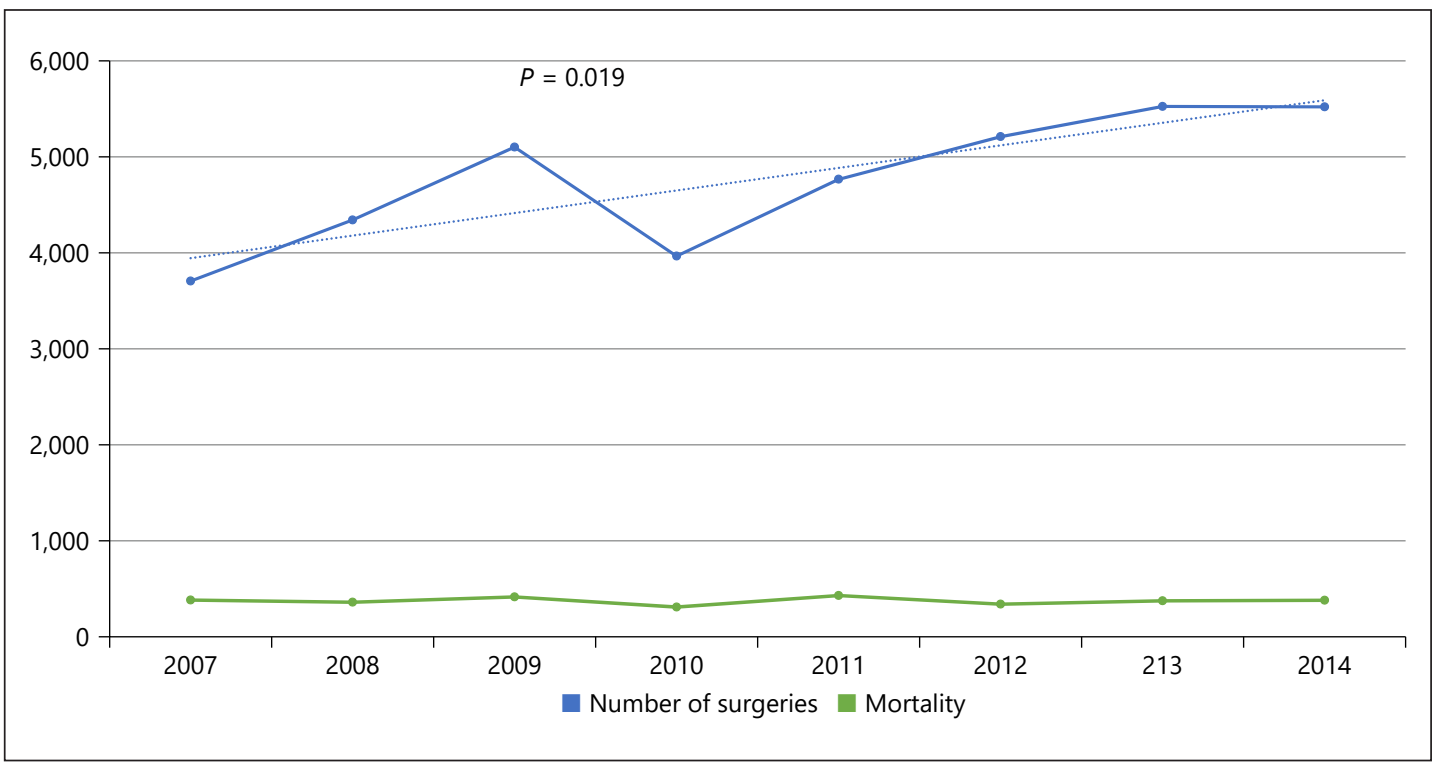

Fig. 3. Trends of tricuspid valve surgery and mortality in native heart.

with a bioprosthetic valve did not experience in-hospital mortality, although this was not statistically significant compared to the other two groups.

OHT patients undergoing TV surgery are more likely to be younger, male, and operated on at an academic hospital. Significant TV pathology at a younger age is most likely driven by repeated endomyocardial biopsies. The gender and hospital demographics are consistent with trends in OHT.

Despite having greater co-morbidities $(\mathrm{CCI} \geq 3)$ and increased LOS, patients in the OHT cohort did not have an increase in mortality during index hospitalization. Added risk of mortality among less sick native heart patients undergoing TV surgery may be driven by the fact that these patients were significantly more likely to have a concomitant surgery compared to patients with OHT. Vassileva et al. [15] have shown concomitant tricuspid replacement to be associated with significantly higher hospital mortality than isolated tricuspid replacement. Nevertheless, this is a very promising finding especially in light of the high expected mortality from significant and untreated TV regurgitation among transplant patients [8].

Prior studies have shown that the proportion of TV surgeries that were repairs have continued to increase over time, which is on par with the most common types of TV surgery in our study cohort $[15,16]$. This practice trend is well founded in that hospital mortality for TV replacement is significantly higher than for TV repair with and without concomitant surgery [15-17]. The best mortality outcomes among OHT patients in our study were among those receiving bioprosthetic TV replacement but this difference did not reach statistical significance. TV replacement with bioprosthetic valve has consistently been shown to render a lower mortality risk compared to mechanical valve among native hearts [15-17]. It has also been reported that native heart patients were more likely to have a concomitant surgery with TV operation. This finding is consistent with the 2014 American Heart Association/American College of Cardiology (AHA/ACC) guidelines that reserve the only class I indication for TV surgery for patients with symptomatic or asymptomatic severe TV regurgitation (stages $\mathrm{C}$ and D) undergoing concomitant left-sided valve surgery [18]. While the 2014 AHA/ACC guidelines do not address distinct indications for TV surgery in OHT patients with TV regurgitation, other literature has stated that while the majority of TV regurgitation that develops following trans- 
plant fails to lead to symptoms significant enough to require repair, in patients that develop medically refractory symptoms, repair and replacement have been deployed $[13,18]$.

There are several important limitations to our analysis. This is a retrospective analysis of registry data from the NIS and is limited to single hospitalizations and does not contain information on longer-term outcomes, including out-of-hospital mortality. We are also limited in our ability to analyze the surgical team's rationale behind choosing the type of surgery for any particular patient. Although errors in ICD-9 coding and documentation are limited in this database, misclassification bias cannot be completely excluded. Additionally, the yearly trend analysis may not be perfectly accurate as the $20 \%$ stratified sample of discharges from the NIS may vary year to year. An additional limitation of our study is that we were unable to adjust for sex mismatch between the donor and the recipient in describing outcomes in the transplant group due to the nature of the database.

In conclusion, surgical intervention for TV regurgitation carries similar mortality during index hospitalization among patients with and without OHT. The number of patients receiving transplant is increasing and patients are living longer after transplant. With this, the incidence of TV regurgitation in this population is expected to increase, making TV surgery an even more relevant treatment modality among OHT patients.

\section{Statement of Ethics}

This project did not have any direct human or animal participants.

\section{Disclosure Statement}

All authors do not have any disclosures pertaining to this submission.

\section{Funding Sources}

No funding was received for this project.

\section{Author Contributions}

Moghniuddin Mohammed and Zubair Shah: study design, statistical analyses, and writing of the manuscript. Aniket S. Rali, Travis Abicht, Nicholas Haglund, and Andrew Sauer: study design and writing of the manuscript. Tyler Buechler, Juwairiya Arshi, Seyed Hamed Hosseini Dehkordi, Jonathan Chandler, and Robert Weidling: writing of the manuscript. Venkat Vuddanda: statistical analyses.

\section{References}

1 Wong RC, Abrahams Z, Hanna M, Pangrace J, Gonzalez-Stawinski G, Starling R, et al. Tricuspid regurgitation after cardiac transplantation: an old problem revisited. J Heart Lung Transplant. 2008 Mar;27(3):247-52.

2 Chan MC, Giannetti N, Kato T, Kornbluth M, Oyer P, Valantine HA, et al. Severe tricuspid regurgitation after heart transplantation. J Heart Lung Transplant. 2001 Jul;20(7):709-17.

3 Davies RR, Russo MJ, Morgan JA, Sorabella RA, Naka Y, Chen JM. Standard versus bicaval techniques for orthotopic heart transplantation: an analysis of the United Network for Organ Sharing database. J Thorac Cardiovasc Surg. 2010;140(3):700-8, 708.e1-2. 
4 Aziz TM, Saad RA, Burgess MI, Campbell CS, Yonan NA. Clinical significance of tricuspid valve dysfunction after orthotopic heart transplantation. J Heart Lung Transplant. 2002 Oct;21(10):1101-8.

5 Berger Y, Har Zahav Y, Kassif Y, Kogan A, Kuperstein R, Freimark D, et al. Tricuspid valve regurgitation after orthotopic heart transplantation: prevalence and etiology. J Transplant. 2012;2012:120702.

6 Marelli D, Esmailian F, Wong SY, Kobashigawa JA, Kwon MH, Beygui RE, et al. Tricuspid valve regurgitation after heart transplantation. J Thorac Cardiovasc Surg. 2009 Jun;137(6):1557-9.

7 Wartig M, Tesan S, Gäbel J, Jeppsson A, Selimovic N, Holmberg E, et al. Tricuspid regurgitation influences outcome after heart transplantation. J Heart Lung Transplant. 2014 Aug;33(8):829-35.

8 Bollano E, Karason K, Lidén H, Dellgren G. How should we manage early tricuspid valve regurgitation after heart transplantation? Int J Cardiol. 2016 Jul;214:191-3.

9 Costanzo MR, Dipchand A, Starling R, Anderson A, Chan M, Desai S, et al.; International Society of Heart and Lung Transplantation Guidelines. The International Society of Heart and Lung Transplantation Guidelines for the care of heart transplant recipients. J Heart Lung Transplant. 2010 Aug;29(8):914-56.

10 Fiorelli AI, Stolf NA, Abreu Filho CA, Santos RH, Buco FH, Fiorelli LR, et al. Prophylactic donor tricuspid annuloplasty in orthotopic bicaval heart transplantation. Transplant Proc. 2007 Oct;39(8):2527-30.

11 Jeevanandam V, Russell H, Mather P, Furukawa S, Anderson A, Grzywacz F, et al. A one-year comparison of prophylactic donor tricuspid annuloplasty in heart transplantation. Ann Thorac Surg. 2004 Sep;78(3):75966.

12 Jeevanandam V, Russell H, Mather P, Furukawa S, Anderson A, Raman J. Donor tricuspid annuloplasty during orthotopic heart transplantation: long-term results of a prospective controlled study. Ann Thorac Surg. 2006 Dec;82(6):2089-95.

13 Kwon MH, Shemin RJ. Tricuspid valve regurgitation after heart transplantation. Ann Cardiothorac Surg. 2017 May;6(3):270-4.

14 R Core Team. R: a language and environment for statistical computing. Vienna: R Foundation for Statistical Computing; 2019. Available from: https://www.r-project.org/index.html.

15 Vassileva CM, Shabosky J, Boley T, Markwell S, Hazelrigg S. Tricuspid valve surgery: the past 10 years from the Nationwide Inpatient Sample (NIS) database. J Thorac Cardiovasc Surg. 2012 May;143(5):1043-9.

16 Kilic A, Saha-Chaudhuri P, Rankin JS, Conte JV. Trends and outcomes of tricuspid valve surgery in North America: an analysis of more than 50,000 patients from the Society of Thoracic Surgeons database. Ann Thorac Surg. 2013 Nov; 96(5):1546-52.

17 Zack CJ, Fender EA, Chandrashekar P, Reddy YN, Bennett CE, Stulak JM, et al. National trends and outcomes in isolated tricuspid valve surgery. J Am Coll Cardiol. 2017 Dec;70(24):2953-60.

18 Nishimura RA, Otto CM, Bonow RO, Carabello BA, Erwin JP 3rd, Guyton RA, et al.; ACC/AHA Task Force Members. 2014 AHA/ACC Guideline for the Management of Patients with Valvular Heart Disease: a report of the American College of Cardiology/American Heart Association Task Force on Practice Guidelines. Circulation. 2014 Jun;129(23):e521-643. 